\title{
Immune regulation and viral diversity as correlates of natural and treatment induced immune control in persistent hepatitis B virus (HBV) infection
}

\author{
Anna Parfieniuk-Kowerda, Jerzy Jaroszewicz, Robert Flisiak \\ Department of Infectious Diseases and Hepatology, Medical University of Białystok, Poland
}

\begin{abstract}
In long-lasting chronic hepatitis B, the phenomenon of cytotoxic CD8 T lymphocytes (CTL) exhaustion and unresponsiveness to HBV-specific stimuli was shown to be crucial for the loss of immune control of the virus and disease activity. There is evidence that Tregs, Th17 cells and Bregs seem to be important in pathogenesis of the immunological dysfunction and loss of HBV-specific activity of cytotoxic CD8 T-cells. Th17-driven immune response was shown to be important in pathogenesis of acute HBV infection and exacerbated chronic hepatitis $B$ along with Th1 response contributing to hepatocellular damage due to proinflammatory activities of Th17-derived cytokines, mainly IL-17A. Treg cell responses may be either beneficial or harmful in HBV infection by limiting liver immunopathology or suppressing protective $\mathrm{T}$ cell responses, thus promoting virus replication and survival. Thus, Treg/Th17 equilibrium seems to be crucial for the outcomes of HBV infection.
\end{abstract}

Key words: HBV, chronic hepatitis B, Th17, Treg, CD8+ cells.

Address for correspondence

Anna Parfieniuk-Kowerda, Department of Infectious Diseases and Hepatology, Medical University of Białystok, 14 Zurawia St., 15-540 Białystok, Poland, e-mail: anna.parfieniuk@gmail.com

\section{Natural history, viral diversity and immune control in hepatitis B virus infection}

Chronic hepatitis B virus (HBV) infection leads to the impaired function of the immune system which enables enhanced viral replication and progression of liver disease to cirrhosis and/or hepatocellular carcinoma. It is interesting why some chronically infected patients are able to control HBV replication at low levels while in others, the virus escapes from immune control and disease progresses. Persistent HBV-infection is a highly dynamic disease which could be categorized in 5 various phases characterized by different HBV-DNA and ALT levels as well as HBeAg-serological status but foremost of different immune activation and viral diversity [1]. The dynamics of disease are especially pronounced in $\operatorname{HBe} \operatorname{Ag}(-)$ hepatitis where significant log-fold fluctuations of HBV-DNA and inflammatory activity are a well-known phenomenon. On the other hand, in some patients, the long-lasting immune control of viral replication can be naturally acquired low replicative phase, inactive HBsAg carriers. Those patients have a very good prognosis with low risk of disease progression and HCC-development. Moreover, it is well known from the natural history of HBV studies that patients achieving sustained immune control have the highest chances of HBsAg loss and seroconversion [2]. Recently, a persistently inactive carrier phase was associated with a low serum concentration of HBsAg, which might reflect a lower concentration of cccDNA as well as the level of HBV-integration in the hepatocytes [3-5]. On the other hand, an overall mechanism driving the immune control of $\mathrm{HBV}$ is not known as immune-based markers of inactive HBsAg carrier phases have not been yet established. One can postulate that the leading mechanism of acquiring viral control in persistent HBV-infection may consist of reconstitution of immune responses, but also the viral heterogeneity may as well play an important role. It has been shown that patients undergoing natural or IFN-induced $\mathrm{HBeAg}$ seroconversion had higher HBV genetic diversity but also the increase in this diversity started to occur just before seroconversion [6]. Some evidence also indicated that specific polymorphisms in genes encoding crucial 
parts of the immune system might be associated with disease progression. For example IL-10 gene promoter polymorphisms, characterized by low capacity for IL-10 production were significantly higher in asymptomatic carriers than in patients with chronic progressive liver disease [7]. Regardless of a lot of evidence pointing towards different aspects of immune responses, the exact immune definition of sustained immune control of HBV is not established. The understanding of sustained immune control might be extremely helpful also for the existing/novel therapies of persistent HBVinfection. It is well known that IFN-therapy offers higher chances of HBeAg as well as HBsAg-loss due to its immunomodulatory features. On the other hand, more widely currently used therapies with nucleotide/nucleoside analogues (NA) are associated with HBsAg-loss only in unique cases, especially in a group of $\mathrm{HBeAg}(-)$ patients. Only limited studies suggest some level of reconstitution of immune responses during NA therapy, for instance telbivudine [8], while it is mainly observed in $\mathrm{HBeAg}(+)$, less prevalent in Europe and the USA. The understanding of mechanisms and markers of persistent immune control of HBV-infection might help optimize combination therapies or develop novel antiHBV therapies aimed at a higher proportion of best therapy end-point which is HBsAg-seroconversion.

\section{Cytotoxic T cells}

In long-lasting chronic hepatitis $\mathrm{B}$, the phenomenon of cytotoxic CD8 T lymphocytes (CTL) exhaustion and unresponsiveness to $\mathrm{HBV}$-specific stimuli was shown to be crucial for the loss of immune control of the virus. However, the role of other "players in this field" like Tregs, Th17 cells and Bregs seems to be important in pathogenesis of immunological dysfunction and loss of HBV-specific activity of cytotoxic CD8 T-cells. There are different pathogenic mechanisms proposed to explain T-cell dysfunction in the course of chronic hepatitis B. Among them, the most important is functional exhaustion due to constant exposure to a high concentration of viral antigens. Several membrane and intracellular molecules are involved in pathogenesis of deterioration of immune responses in persistent $\mathrm{HBV}$ infection, including PD1 (Programmed Death 1 receptor), Tim-3 (T cell immunoglobulin mucin-3), CD57, CTLA-4 (Cytotoxic T-Lymphocyte Antigen 4, CD152), 2B4, BTLA. There is evidence that a high expression of PD-1 on T cells induces $\mathrm{T}$ cells inactivation, hence blocking PD-1 results in restoring $\mathrm{HBV}$-specific immune responses in CD8 T cells [9-11]. Another negative regulatory molecule Tim-3 was found to be highly upregulated on T cells, especially HBV-specific CD8 T cells, in patients with chronic hepatitis $\mathrm{B}$ as compared to healthy. A higher Tim-3 expres- sion on CD8 T cells was associated with lower ability to produce IFN- $\gamma$ and TNF- $\alpha$ in response to HBV-specific stimuli and tendency to undergo apoptosis. Moreover, blocking Tim-3 allowed to restore HBV-specific CD8 T-cell responses. Dual blockade of Tim-3 and PD- 1 receptors resulted in augmentation of $\mathrm{HBV}$-specific CD8 T cell immune responses [12]. One more indicator of functional inactivity of CD8 T cells appeared to be surface CD57 protein. There is evidence that CD57 expression is characteristic of terminally differentiated CD8 T cell and is associated with proliferative inability and replicative senescence. These cells are more prone to undergo apoptosis due to a higher expression of Fas and FasL, but also are able to secrete large quantities of IFN- $\gamma$ upon TCR (T cell receptor) stimulation [13-15]. CTLA-4 receptor is upregulated on activated CD8 T cells upon their stimulation and transmits a regulatory inhibitory signal. In chronic HBV infection, CTLA- 4 was found to be upregulated on $\mathrm{CD} 8 \mathrm{~T}$ cells and correlated with the serum HBV DNA level [16]. 2B4 receptor (CD244) is a member of the signaling lymphocyte activation molecule (SLAM) family of immune cell receptors present mainly on NK and CD8 T cells. 2B4 exhibited an activating function when expressed at low levels, while generated an inhibitory signal when expressed at high levels. In persistent $\mathrm{HCV}$ infection 2B4 was upregulated on $\mathrm{HCV}$-specific CD8 T cells [17]. Recent data suggest that 2B4 is involved in pathogenesis of CD8 T cells exhaustion during chronic HBV infection [18].

\section{Role of interleukin-10}

A balance of immune activation is to some extent orchestrated by regulatory cells. Interleukin-10 is a key regulator of innate and adaptive immune responses by limiting T-cell activation and suppressing proinflammatory responses in tissues leading to impaired pathogen control and reduced immunopathology at the same time [19]. In acute hepatitis B or hepatic flares during chronic HBV infection, IL-10 was shown to moderate immune responses and ameliorate hepatic damage [20]. Though, this probably might be followed by increased viral replication. On the other hand, diminished immune regulation might allow to rebuild functional immune response in late phases of chronic hepatitis B. Our preliminary results in HBeAg-negative chronic hepatitis $B$ patients have shown that low serum IL-10 and HBsAg levels were associated with a sustained inactive HBsAg carrier phase in the longterm follow-up (median follow-up - 31 months). Interestingly, our data suggested that a single measurement of IL-10 and HBsAg might discriminate between a stable low replicative carrier and patients with fluctuating (thus dynamic) disease [21]. In the healthy, 
main sources of IL-10 are predominantly monocytes. Interestingly, in persistent viral infections, also chronic hepatitis B, T and B regulatory cell were identified as important sources of large quantities of IL-10.

\section{Regulatory T cells}

Regulatory T cells (Treg) are a heterogeneous population of $\mathrm{CD} 4+$ cell exerting negative regulatory effects on immune responses, which have the ability to secrete IL-10 (Tr1 subset) or TGF- $\beta$ (Th3). The characteristic feature of regulatory $\mathrm{CD} 4 \mathrm{~T}$ cells is a high expression of CD25 molecule, low CD127 expression and induction of Foxp3 transcription factor. Treg cells are important components of negative regulation of immune responses. Deregulation of this system leads to exaggerated, often destructive immune (mainly Th1, TH17) activation leading to tissue damage. On the contrary, augmented Treg responses lead to impaired eradication of pathogens and might have a role in pathogenesis of human malignancy. Treg cell responses may be either beneficial or harmful in HBV infection by limiting liver immunopathology or suppressing protective $\mathrm{T}$ cell responses thus promoting virus replication and survival [22].

\section{Regulatory B cells}

Recent reports suggest that regulatory B cells suppress HBV-specific CD8+ T cell responses thus dimi- nishing antiviral immune response. The pathogenic mechanism involved modulation of antiviral response by B-cells derived IL-10, since blockade of IL-10 restored functionality of CD8+ T-cells. Moreover, the frequency of IL-10 producing B-cells was higher parallel to the aggravation of disease activity in chronic hepatitis B. The subset of B-cells producing IL-10 was identified as $\mathrm{CD} 24^{\mathrm{hi}} \mathrm{CD} 38^{\mathrm{hi}}$ immature B-cells. Authors suggest that increased IL-10 production is a protective mechanism that aims to ameliorate hepatic damage during flares with CD8 T-cells suppression as a bystander effect [20].

\section{Interleukin-17 producing helper T cells - Th17 cells}

Th17 cells were distinguished from other $\mathrm{T}$ cells mainly on the basis of IL-17 production and presence of ROR- $\gamma \mathrm{t}$ transcription factor. Th17 driven immune response was shown to be important in pathogenesis of acute HBV infection and exacerbated chronic hepatitis $B$ along with Th1 response contributing to hepatocellular damage due to proinflammatory activities of Th17-derived cytokines, mainly IL-17A [23]. The crucial role of Th17 cells was confirmed in various inflammatory and autoimmune diseases as Th17 activation leads to cellular/tissue damage through proinflammatory cytokine milieu: IL-17, IL-22, IL-21. However, recent reports are equivocal as to the role of Th17 cells in regulation of immune responses since the regulation of the

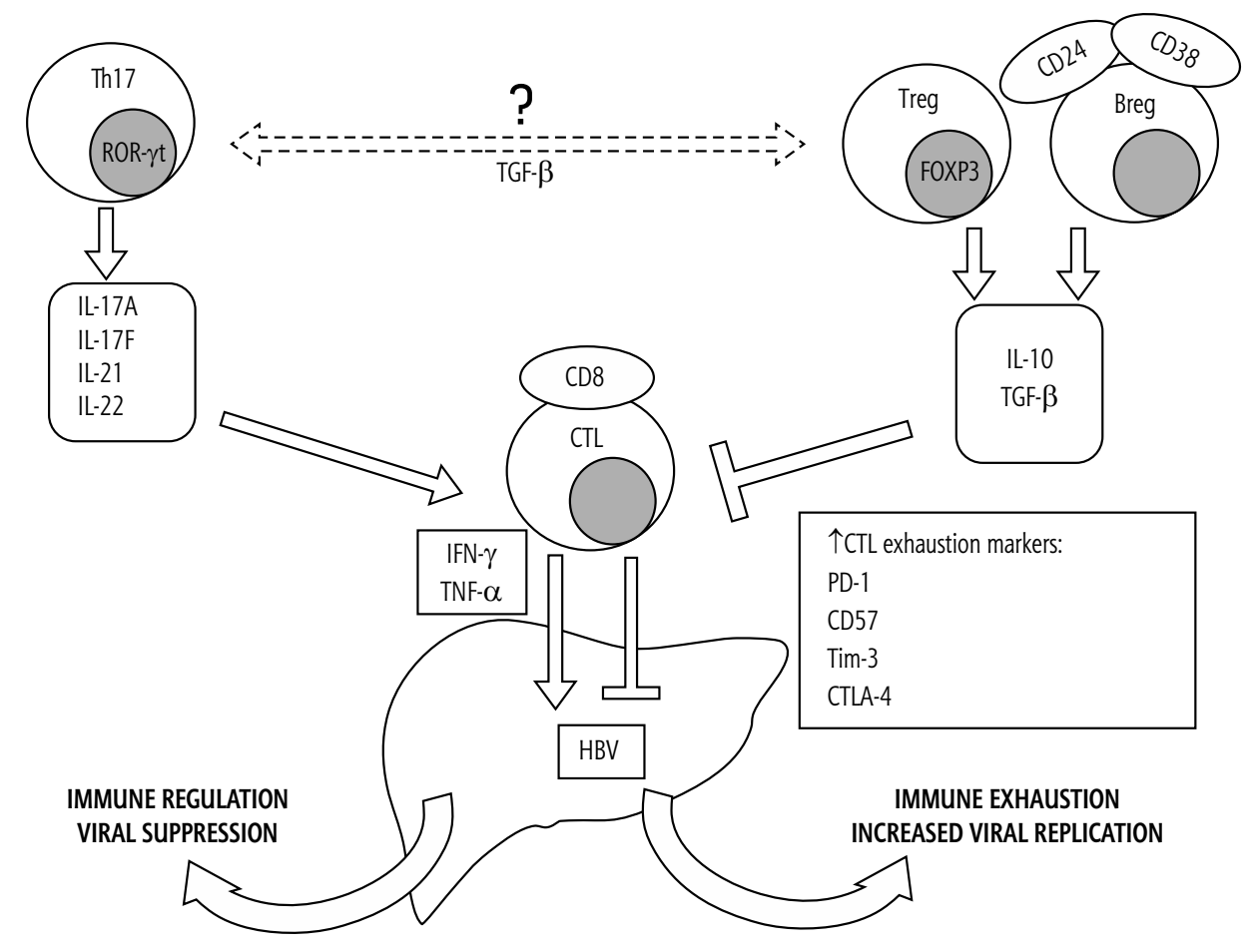

Fig. 1. Regulatory immune responses and Th17 responses in chronic hepatitis B 
production of Th17-cells cytokines - IL-17A, IL-17F, as well as IL-21 and IL-22 is distinct and results from different chemokine stimuli [24]. Our preliminary analysis of Th17 serum cytokine profile in HBeAg-negative persistent HBV infection has shown that serum IL-17F was significantly lower in HBV infection compared to controls. Furthermore, in chronic HBV infection, a higher serum concentration of IL-17F was associated with immune control of infection [25]. Recent data demonstrated that in acute HCV infection, Th-17 derived IL-21 was shown to prevent CD8 $\mathrm{T}$ cell exhaustion by counteracting Tim3/Gal-9 interactions [26].

The balance between regulatory immune responses and Th17 responses was shown to influence the progression of HBV infection (Fig. 1) [27]. Moreover, there is evidence that a decreased ratio of Tregs to Th17 cells as a result of antiviral treatment might indicate control of HBV infection [28]. Thus, Treg/Th17 equilibrium seems to be crucial for the outcomes of HBV infection.

\section{Disclosure}

Authors report no conflict of interest.

\section{References}

1. Thompson A, Locarnini S, Visvanathan K. The natural history and the staging of chronic hepatitis B: time for reevaluation of the virus-host relationship based on molecular virology and immunopathogenesis considerations? Gastroenterology 2007; 133: 1031-1035.

2. Fattovich G, Bortolotti F, Donato F. Natural history of chronic hepatitis B: special emphasis on disease progression and prognostic factors. J Hepatol 2008; 48: 335-352.

3. Brunetto MR, Oliveri F, Colombatto P, et al. Hepatitis B surface antigen serum levels help to distinguish active from inactive hepatitis B virus genotype D carriers. Gastroenterology 2010; 139: 483-490.

4. Nguyen T, Thompson AJ, Bowden S, et al. Hepatitis B surface antigen levels during the natural history of chronic hepatitis $\mathrm{B}$ : a perspective on Asia. J Hepatol 2010; 52: 508-513.

5. Jaroszewicz J, Calle Serrano B, Wursthorn K, et al. Hepatitis B surface antigen ( $\mathrm{HBsAg}$ ) levels in the natural history of hepatitis B virus (HBV)-infection: a European perspective. J Hepatol 2010; 52: 514-522.

6. Lim SG, Cheng Y, Guindon S, et al. Viral quasi-species evolution during hepatitis Be antigen seroconversion. Gastroenterology 2007; 133: 951-958.

7. Miyazoe S, Hamasaki K, Nakata K, et al. Influence of interleukin-10 gene promoter polymorphisms on disease progression in patients chronically infected with hepatitis B virus. Am J Gastroenterol 2002; 97: 2086-2092.

8. Wursthorn K, Jung M, Riva A, et al. Kinetics of hepatitis B surface antigen decline during 3 years of telbivudine treatment in hepatitis B e antigen-positive patients. Hepatology 2010; 52: 1611-1620.

9. Zhang Z, Zhang JY, Wherry EJ, et al. Dynamic programmed death 1 expression by virus-specific CD8 T cells correlates with the outcome of acute hepatitis B. Gastroenterology 2008; 134: 1938-1949.
10. Fisicaro P, Valdatta C, Massari M, et al. Combined blockade of programmed death-1 and activation of $\mathrm{CD} 137$ increase responses of human liver T cells against HBV, but not HCV. Gastroenterology 2012; 143: 1576-1585.

11. Fisicaro P, Valdatta C, Massari M, et al. Antiviral intrahepatic T-cell responses can be restored by blocking programmed death-1 pathway in chronic hepatitis B. Gastroenterology 2010; 138: 682-693.

12. Nebbia G, Peppa D, Schurich A, et al. Upregulation of the Tim-3/ galectin-9 pathway of $\mathrm{T}$ cell exhaustion in chronic hepatitis $\mathrm{B}$ virus infection. PLoS One 2012; 7: e47648.

13. Le Priol Y, Puthier D, Lecureuil C, et al. High cytotoxic and specific migratory potencies of senescent CD8+ CD57+ cells in HIV-infected and uninfected individuals. J Immunol 2006; 177: 5145-5154.

14. Papagno L, Spina CA, Marchant A, et al. Immune activation and CD8+ T-cell differentiation towards senescence in HIV-1 infection. PLoS Biol 2004; 2: E20.

15. Brenchley JM, Karandikar NJ, Betts MR, et al. Expression of CD57 defines replicative senescence and antigen-induced apoptotic death of CD8+ T cells. Blood 2003; 101: 2711-2720.

16. Schurich A, Khanna P, Lopes AR, et al. Role of the coinhibitory receptor cytotoxic $\mathrm{T}$ lymphocyte antigen- 4 on apoptosis-Prone CD8 T cells in persistent hepatitis B virus infection. Hepatology 2011; 53: 1494-1503.

17. Schlaphoff V, Lunemann S, Suneetha PV, et al. Dual function of the NK cell receptor 2B4 (CD244) in the regulation of HCV-specific CD8+ T cells. PLoS Pathog 2011; 7: e1002045.

18. Bengsch B, Seigel B, Blum HE, Thimme R. Differenzial functional enhancement of HBV-specific CD8 + T cell upon inhibitory receptor blockade. Z Gastroenterol 2013; 51: P_5_04.

19. Couper KN, Blount DG, Riley EM. IL-10: the master regulator of immunity to infection. J Immunol 2008; 180: 5771-5777.

20. Das A, Ellis G, Pallant C, et al. IL-10-producing regulatory B cells in the pathogenesis of chronic hepatitis B virus infection. J Immunol 2012; 189: 3925-3935.

21. Jaroszewicz J, Parfieniuk-Kowerda A, Swiderska M, Rogalska M, Lapinski TW, Flisiak R. Low serum IL-10 and HBsAg concentrations are associated with long therm immune control in persistent HBV infection. Hepatology 2013; 58: 224A-225A.

22. Stoop JN, van der Molen RG, Baan CC, et al. Regulatory T cells contribute to the impaired immune response in patients with chronic hepatitis B virus infection. Hepatology 2005; 41: 771-778.

23. Zhai S, Zhang L, Dang S, et al. The ratio of Th-17 to Treg cells is associated with survival of patients with acute-on-chronic hepatitis B liver failure. Viral Immunol 2011; 24: 303-310.

24. Melton AC, Melrose J, Alajoki L, et al. Regulation of IL-17A production is distinct from IL-17F in a primary human cell co-culture model of T cell-mediated B cell activation. PLoS One 2013; 8: e58966.

25. Parfieniuk-Kowerda A, Jaroszewicz J, Swiderska M, et al. Higher serum concentration of IL-17F is associated with immune control of HBV infection. J Hepatol 2013; 58 (S1): S222.

26. Kared H, Fabre T, Bedard N, et al. Galectin-9 and IL-21 mediate cross-regulation between Th17 and Treg cells during acute hepatitis C. PLoS Pathog 2013; 9: e1003422.

27. Li J, Qiu SJ, She WM, et al. Significance of the balance between regulatory $\mathrm{T}$ (Treg) and $\mathrm{T}$ helper 17 (Th17) cells during hepatitis B virus related liver fibrosis. PLoS One 2012; 7: e39307.

28. Zhang JY, Song CH, Shi F, et al. Decreased ratio of Treg cells to Th17 cells correlates with HBV DNA suppression in chronic hepatitis B patients undergoing entecavir treatment. PLoS One 2010; 5: e13869. 